\title{
The LEDA galaxy distribution
}

\section{Maps of the local universe ${ }^{\star}$}

\author{
H. Courtois ${ }^{1}$, G. Paturel ${ }^{1}$, T. Sousbie ${ }^{1}$, and F. Sylos Labini ${ }^{2}$ \\ ${ }^{1}$ Centre de Recherche Astronomique de Lyon (CRAL), 9 avenue Charles André, 69561 Saint Genis Laval Cedex, France \\ e-mail: courtois@obs.univ-lyon1.fr \\ ${ }^{2}$ Laboratoire de Physique Théorique, Université Paris XI, Bâtiment 210, 91405 Orsay Cedex, France
}

Received 17 November 2003 / Accepted 1 March 2004

\begin{abstract}
To investigate the properties of large-scale structures of galaxies in the universe, we present an analysis of their spatial distribution at $z<0.033$. We used the LEDA extragalactic database containing over 1 million galaxies covering the all-sky and the SDSS data included in the public release DR1, yielding a sample of around 134000 galaxies having a measured redshift in two survey areas representing 690 sq. degrees. The results of the study are 2D, 3D maps and magnitude number counts of galaxies, drawn from $B$-band samples.
\end{abstract}

Key words. cosmology: large-scale structure of Universe

\section{Introduction}

Angular and radial maps of the Local Universe are the first step for astronomers to investigate the nature of large-scale structures observed in the galaxy distribution. An early discovery of a large-scale structure was the "great wall" of galaxies, in the CfA1 redshift survey (Geller \& Huchra 1989; Huchra \& Burg 1992). Building on this promising technique for tracing 3D structures, deeper redshift surveys including CfA2 (Huchra et al. 1995), IRAS, LCRS, 2dF (Colless et al. 2001), and SDSS (Abazajian et al. 2003) all show new more extensive and complex structures such as walls, chains, voids and superclusters. A related approach to quantify the complexity of the galaxy distribution is to construct the galaxy number counts in mag, $N(m)$ and in distance, $N(r)$, using statistical indicators: such as spatial and angular two-point correlation functions. Combining these approaches, the physical interpretation of these counts leads to the understanding of the nature of large-scale structure and to its cosmological implications for the models of formation of galaxies. Much attention has been focused on the determination of the scale of homogeneity in the universe. It is not a trivial concern; some statistical indicators are interpreted as showing the scale of homogeneity to be as small as $15 \mathrm{Mpc}$, while the new redshift surveys show extended structures larger than $100 \mathrm{Mpc}$, dwarfing even cluster-sized stucture. In the present paper, we will not directly address this issue, but instead focus on the nearby

* The 3 avi files are only available in electronic form at http://www. edpsciences.org
Universe, creating a global picture for the redshift and number count distribution of galaxies.

The paper is organized as follows: Sect. 2 presents the data, the completness studies, the number counts and the construction of volume-limited subsamples; Sect. 3 shows the 2D and 3D maps of the Local Universe in various coordinate systems and preliminary comparison with SDSS DR1 and Sect. 4 gives our conclusions.

\section{Building the samples}

\subsection{The LEDA database}

The Lyon-Meudon Extragalactic Database (LEDA) was the first database created to collect all published measurements on galaxies related to studies of the structure and kinematics of the Local Universe. For this purpose, the database is limited to galaxies closer than $60000 \mathrm{~km} \mathrm{~s}^{-1}$. It was created by Georges Paturel in 1983 (see Paturel et al. 1997) relaying the work of Gérard de Vaucouleurs and his famous RC1 and RC2 catalogs (de Vaucouleurs et al. 1976). The RC3 catalog (de Vaucouleurs et al. 1991) was made using the LEDA database. This archive has stimulated a large and varied number of studies, over 100 refereed papers, that address large-scale structure and peculiar velocity flows. In the year 2000, the database galaxy number was increased to over 1 million. For the 20th anniversary of LEDA in 2003, completely new studies of the all-sky Local Universe are possible. One should insist on the fact that there is no other possibility to work with all-sky homogeneized magnitudes in a volume of $3 \times 10^{6} \mathrm{Mpc}^{3}$. This is true for B selected surveys, however 
Table 1. Data in LEDA in 2003.

\begin{tabular}{ll}
\hline \hline Data & Number \\
\hline Galaxies & 1316143 \\
$B$ magnitudes & 929815 \\
Radial velocities & 213446 \\
HI width measurements & 51621 \\
\hline
\end{tabular}

the 2MASS survey was designed precisely to be a homogenized, all-sky, uniform survey of galaxies (as well as the Milky Way). In the NIR, 2MASS is more complete than the optical surveys since it penetrates the Zone of Avoidance. One can see the spectacular results in the all-sky data release (XSC: extended source catalog, see Cutri et al. 2003; Jarrett et al. 2000).

In the paper we use $H_{0}=100 \mathrm{Mpc} \mathrm{km}^{-1} \mathrm{~s}^{-1}$. The non-Betaversion of the DR1 of SDSS was released in June 2003, and is simultaneously employed to discern the large-scale structures in a slice of $600 \mathrm{Mpc}$ depth. The extinction correction on LEDA $B$ magnitudes has been made according to Schlegel et al. (1998) since 2002 in LEDA. Hereafter we refer to these $\mathrm{B}$ total magnitudes corrected for galactic extinction as $B$ tc magnitudes.

\subsection{Completness studies}

To build representative maps of the Local Universe, we should first test the completness of the samples. In the case of the LEDA database, we compare the number counts in apparent magnitude in the $B$ band. The magnitudes are corrected for galactic extinction are noted hereafter $B$ tc. In the database there are about 930000 galaxies with a measured $B$ magnitude (hereafter the photometric catalog), 125685 of them have also a measured redshift (hereafter the redshift catalog).

Before testing the completness of the catalogs available in LEDA, we exclude the Zone of Avoidance $(-15 \mathrm{deg}<$ galactic latitude $<15 \mathrm{deg}$ ). The Zone is very incomplete, so one would expect many galaxies to be missing (as well as galaxy clusters and superclusters, including the Great Attractor). Galactic extinction is accounted for in LEDA Btc magnitudes, but that only works for the low extinction cases $(A v<1)$. In the Galactic Plane the extinction is very patchy and is not always well traced with the Schlegel maps. Thus, it is better for our studies of completness to exlude this zone.

In Fig. 1 we plot the magnitude number counts of the two subsamples: the "photometric" one and the "redshift" one. There is a linear growth of the number of galaxies with the tested volume. However the slopes are not following the $0.6 \mathrm{~m}$ expected if the distribution of galaxies were homogeneous in the Local universe. For the photometric sample, the slope is 0.52 and for the redshift one it is 0.51. These slopes are measured only in the part where the incompleteness can be neglected. If we suppose that the photometric catalog is complete, then the redshift one contains $84 \%$ of the galaxies at $B \mathrm{tc}=14.5$ and $90 \%$ at $B \mathrm{tc}=11$.

In the literature there are various interpretations for the values of these slopes: local underdensity, fractal behaviour below

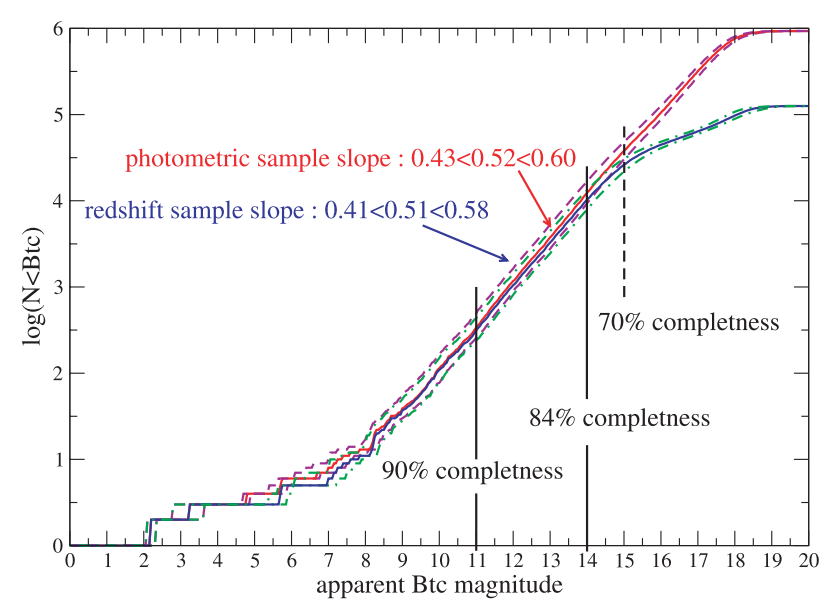

Fig. 1. Magnitude number counts for LEDA: decimal logarithm of the cumulative number of observed galaxies versus the extinction-corrected $B$ total magnitude $(B \mathrm{tc})$. The zone of avoidance $(-15 \mathrm{deg}<$ galactic latitude $<15 \mathrm{deg}$ ) has been cut out of the catalog, and its completness is not tested here. There is a clear change of slope at $B \mathrm{tc}=14.5$ in the redshift catalog and at $B \mathrm{tc}=17$ in the photometric one. These indicate the limit in magnitude where the catalogs reach incompletness. The red and green dashed lines show the smallest and largest error bars on the slope due to the error measurements on Btc. Their slopes corresponds to the largest and smallest values given for the mean slope.

the scale of homogeneity, incompletness of the low surface brightness galaxies. The studies of the SDSS data which are much deeper, showing a slope of 0.45 as we will see in a following paper, tends to cancel out the local underdensity explanation, or at least extend this local underdensity to scales of $600 \mathrm{Mpc}$ depth. Recent surveys dedicated to low surface brightness galaxies tend to show that those galaxies are not present in large-scale voids nor in our vicinity. We can then neglect their effect on the magnitude number counts slopes. The departure from linearity of these slopes gives us the limit of completness of the samples. It is $B t c=14.5$ for the redshift catalog and $B t c=17$ for the photometric one. Knowing those limits, we will be able to define volume limited samples.

We did various tests to quantify the error on the magnitude number count slope due to the errors on the magnitudes. One can find previous studies of LEDA galaxy number counts in Di Nella et al. (1996) and Paturel et al. (1994). It is not the purpose of this paper to discuss deeply those counts, here we use them only in order to build representative samples of galaxies to allow a construction of a set of map. We measured the slope in 3 cases taking into account the error on the magnitudes which is respectively zero, equal to the real error and the double of the real error. In this manner we check that the error has little influence on the slope of the magnitude number counts. In Fig. 1 we give the smallest and the largest values of the slopes we could obtain when accounting for the maximum of the error bars on each value of the magnitude.The errors on the magnitudes in LEDA are so large that the error on the slope is about 0.09 , so we can not totally exclude a slope of 0.6 , in particular for the photometric catalog. 


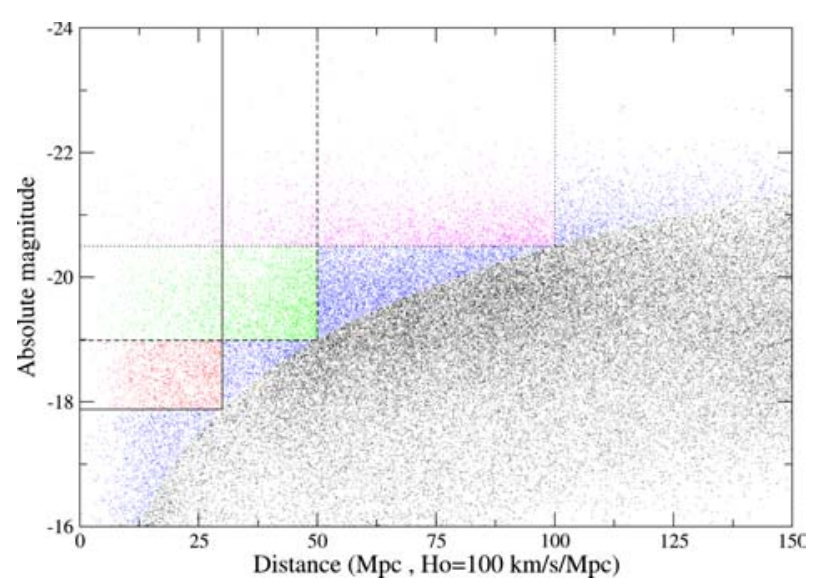

Fig. 2. Absolute magnitude limits for the volume limited subsamples.The redshift catalog shown in black contains 125685 galaxies. The blue points, 16906 sources, denote a Btc less than 14.5 selection. The red points, 2504 sources, denote a Volume Limited (VL) cut at $30 \mathrm{Mpc}$; the green points, 4434 sources, denote a VL cut at $50 \mathrm{Mpc}$; purple points, 2814 sources, denote a VL cut at $100 \mathrm{Mpc}$.

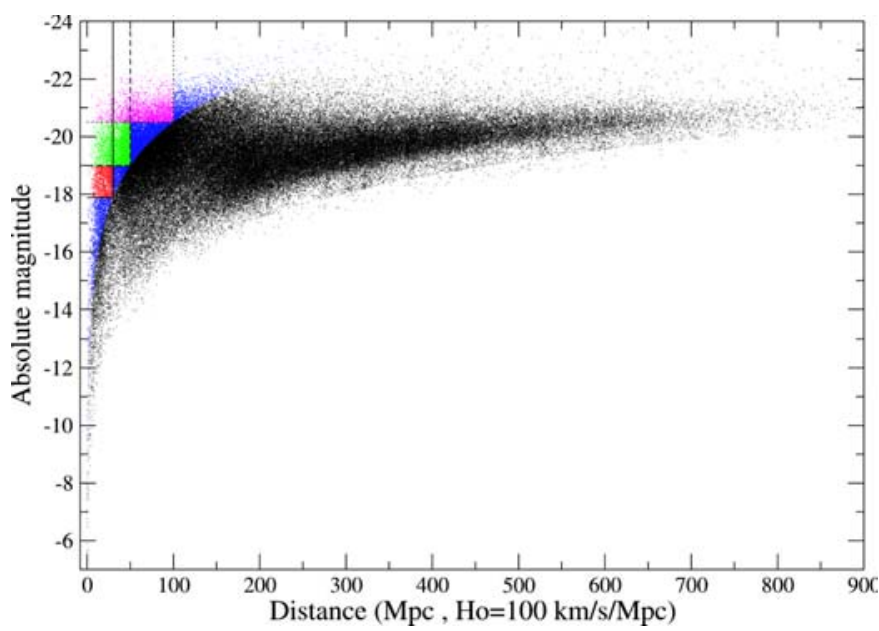

Fig. 3. Completness limits for the volume limited subsamples. The colours are the same as in Fig. 2.

\subsection{Volume limited subsamples}

In Figs. 2 and 3 we can see how the cut in apparent magnitude $B$ tc $<14.5$ affects the redshift samples. From 125685 galaxies (in black) we have now (in blue) only 16906 galaxies left. Those samples have been observed with selection in apparent magnitude, thus they contain a wide variety of galaxies. The distribution in absolute magnitude within these samples is incomplete. They contain dwarf galaxies, normal galaxies and giant galaxies, but each of these classes is not complete. The apparent magnitude surveys do not contain all dwarf, all normal and all giant galaxies. To select only galaxies that are statistically representative, ie, having the same absolute magnitude, we apply a cut in distance and in absolute magnitude. We build three volume-limited subsamples at 30 (red), 50 (green) and 100 (purple) Mpc. The number of galaxies left in those samples can be read from Table 2. As we go deeper with the volume limited samples, we select only luminous galaxies. However, one can see in Fig. 3 that the resulting
Table 2. Volume limited subsamples.

\begin{tabular}{ccc}
\hline \hline Lim. distance & Lim. abs. magnitude & Number of gal. \\
\hline $30 \mathrm{Mpc}$ & -17.9 & 2504 \\
$50 \mathrm{Mpc}$ & -19 & 4434 \\
$100 \mathrm{Mpc}$ & -20.5 & 2814 \\
\hline
\end{tabular}

number of galaxies in the deepest volume limited subsample at $100 \mathrm{Mpc}$ contains only about $2 \%$ of the initial redshift catalog of 125685 galaxies.

\section{Maps of the local universe}

\section{1. $2 D$ maps}

In Figs. 4-6 we show angular maps of the Local Universe. They are Flamsteed projections in supergalactic coordinates. This method of representing an angular catalog has the advantage of beeing an "equal area projection", thus conserving the projected areas. However, as with any projection, there are deformations effects near the poles (top and bottom of the figures) and all along the sides.

In Fig. 4, the catalog of all galaxies having a measured $B$ magnitude is shown in black. The galaxies having a magnitude brighter than 14.5 are in red. We can see that only this first selection step allows us to distinguish between real structures and incompletness effects. For example near the zone of avoidance, in the center of the figure, one can see two vertical bands corresponding to the Las Campanas Redshift Survey (LCRS). These bands disappear after the cut in apparent magnitude, ie this survey has a completeness limit much deeper than the completeness limit of the composite all-sky collection of redshift surveys in LEDA.

One structure that can be seen very clearly in Fig. 5 is a kind of wave in the structures appearing in the upper left part of the figure and in the lower right. Such a deformation in a 2D map can be due to a large scale plane of galaxies in the 3D distribution. This wave passes through Perseus-Pisces, Pavo-Indus, Hydra-Centaurus, Virgo and Coma superclusters. It is known and was described as the hypergalactic plane in Di Nella \& Paturel (1994). The hypergalactic plane is not equivalent to the supergalactic plane. The supergalactic plane describes the local supercluster (LSC) plane at a shorter distance from the observer. The hypergalactic plane contains the local supercluster, as the Virgo cluster (the center of the LSC) is lying on this plane extending to larger scale.

\subsection{D maps}

After inspection of the galaxy distribution using 2D maps, it is constructive to view the allsky maps with a 3D projection using the VL redshift sample. Here we project the supergalactic coordinates and redshift into an $X Y Z$ volume; Figs. 7 and 8. The latest largest all-sky 3D maps where published almost ten years ago (Di Nella \& Paturel 1994, 1995; Di Nella et al. 1997; Amendola et al. 1997); this paper 


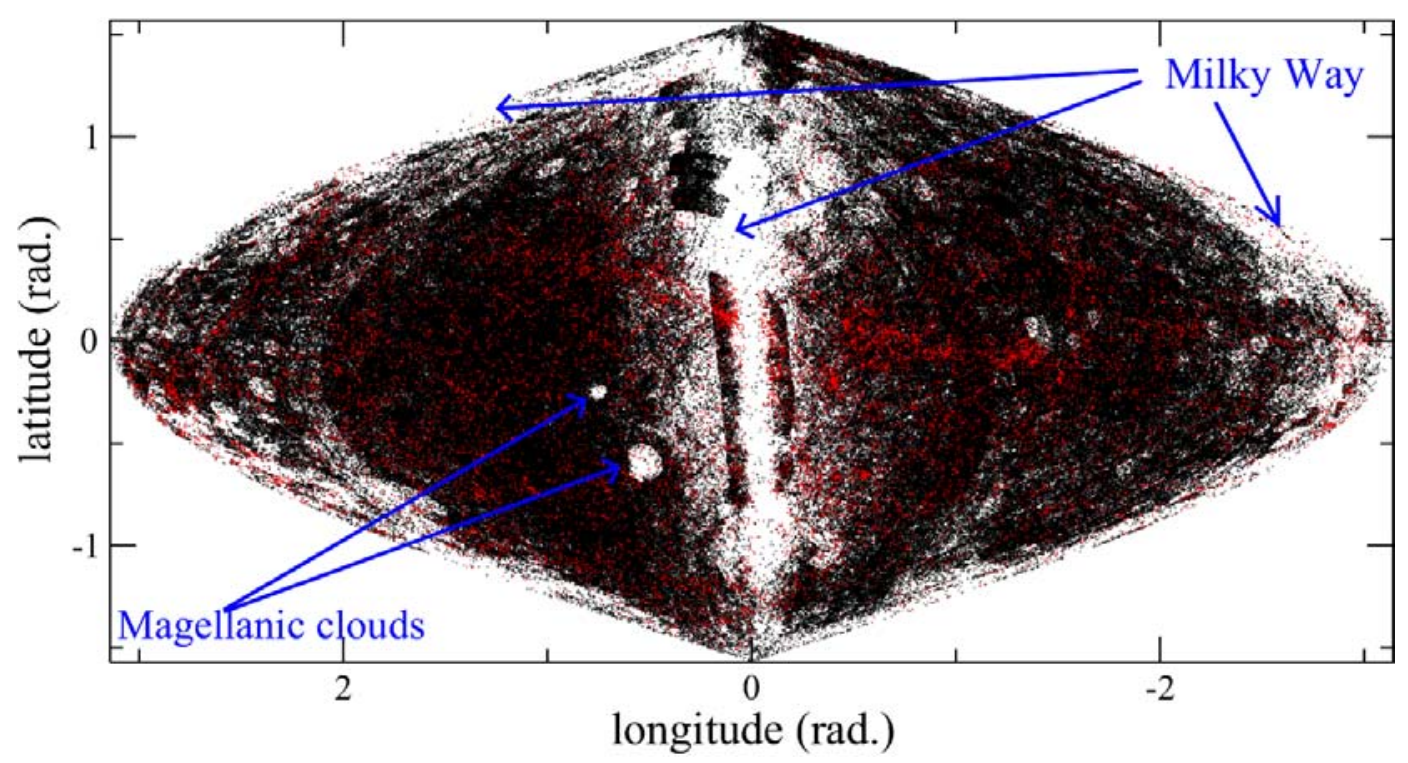

Fig. 4. 2D map of the Local Universe: in black: all galaxies (983 300) with a measured $B$ magnitude in LEDA and in red: the magnitude limited sample: $B \mathrm{tc} \leq 14.5$ (21 859 galaxies). Flamsteed equal area projection in supergalactic coordinates.

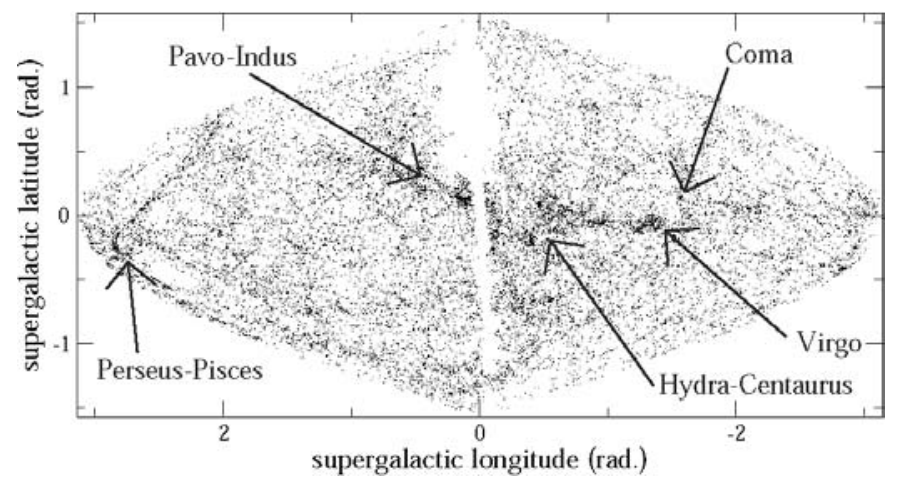

Fig. 5. 2D map of the Local Universe: all galaxies (21 859) where $B$ tc $<14.5$ in LEDA are the black dots. Major superclusters in our neighborhoods are shown. Flamsteed equal area projection in supergalactic coordinates.

improves the data number and more importantly the quality of the magnitudes used.

\subsection{Preliminary comparison with SDSS DR1}

To go further in the mapping of the Local Universe, every astronomer working on large-scale structures has been waiting for the Sloan Digitized Sky Survey (SDSS). These data are of first order quality because they provide the magnitudes in 5 bands, helpful in quantifying the evolution effects on the number counts. In Fig. 9, one can see the first public datarelease (DR1) of the Sloan Digitized Sky Survey (SDSS; Abazajian et al. 2003). The first amazing fact to note is the continuity of bubbles, walls and void sequences up to a scale 6 times larger than the one we just discussed: up to $z=0.2$. The second point is the structure size: we can see a chain (or a wall) of galaxies extending about $300 \mathrm{Mpc}$. As a comparison, we ploted the CfA2 (Huchra \& Burg 1992; Huchra et al. 1995) redshift survey containing the Great Wall

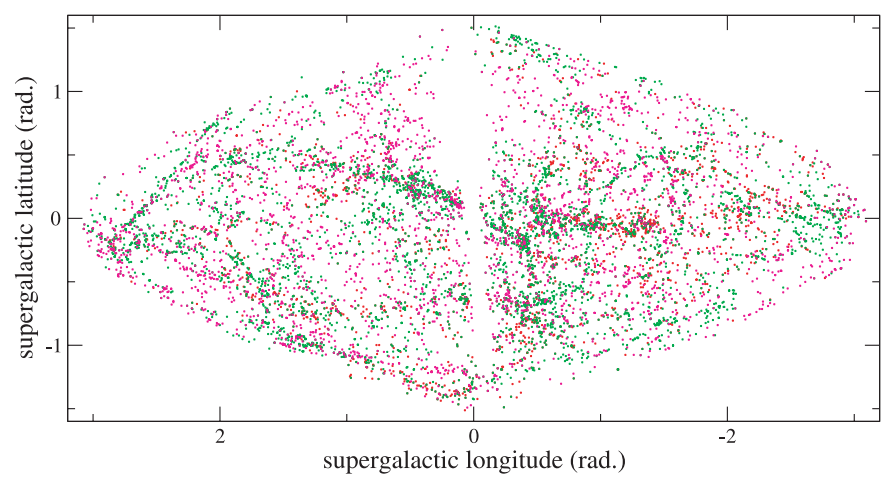

Fig. 6. 2D map of the Local Universe: volume-limited subsamples. Flamsteed equal area projection in supergalactic coordinates. One can see that the different VL subsamples do not trace the same structures. Colour code is: red VL30 Mpc, green VL50 Mpc, purple: VL100 Mpc.

of galaxies, which is about $100 \mathrm{Mpc}$ long. We are now seeing a structure 10 times larger than the Great Wall. The $2 \mathrm{dF}$ redshift survey (Colless et al. 2001) probed a region with comparable depth to SDSS, revealing large-scale structure with equally impressive detail and complexity. Combined, these redshift surveys suggest structures as large as $300 \mathrm{Mpc}$ in extent, probably associated with "walls" of galaxy clusters.

To help visualize structure in the Local Universe, we have created 3 animated renderings of the LEDA and SDSS redshift samples in $X Y Z$ space. The first, LEDA.avi, shows all LEDA galaxies with redshifts measurements, the second, LEDA145.avi, shows the LEDA galaxies brighter than $14.5 \mathrm{mag}$ in Btc, the third, LEDASDSSDR1.avi, combines the LEDA redshifts with those of SDSS (DR1). The movies show the galaxy distribution in 3D space, rotated about each axis separately. For the LEDA/SDSS movie, the LEDA points are colored blue, while the SDSS points are colored yellow. Note the much greater depth, but more patchy coverage, of the SDSS sample compared to the LEDA redshift sample. 


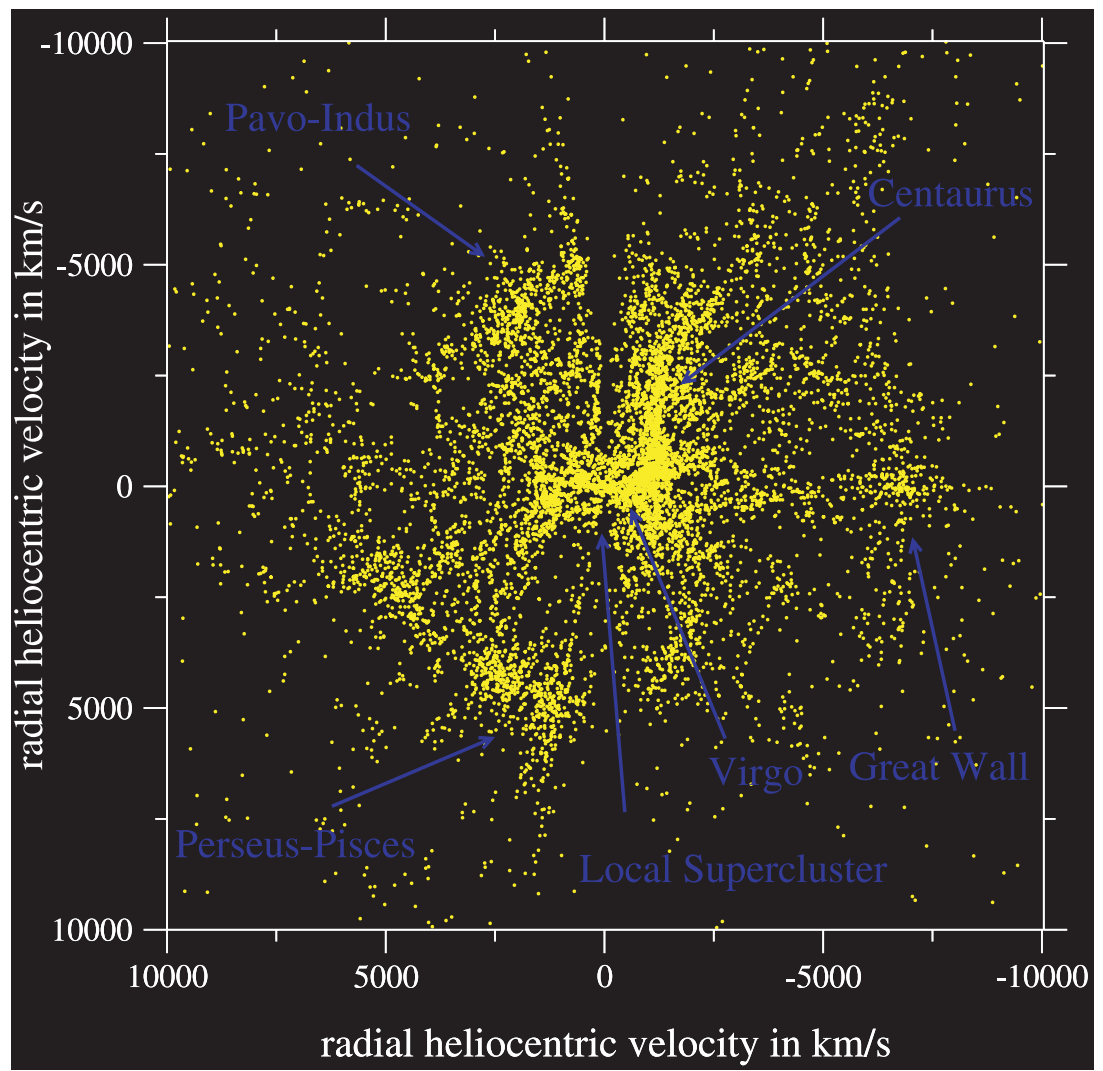

Fig. 7. View face-on of the $X Y$ plane in supergalactic coordinates, slice of $50 \mathrm{Mpc}$ along the $Z$ axis.

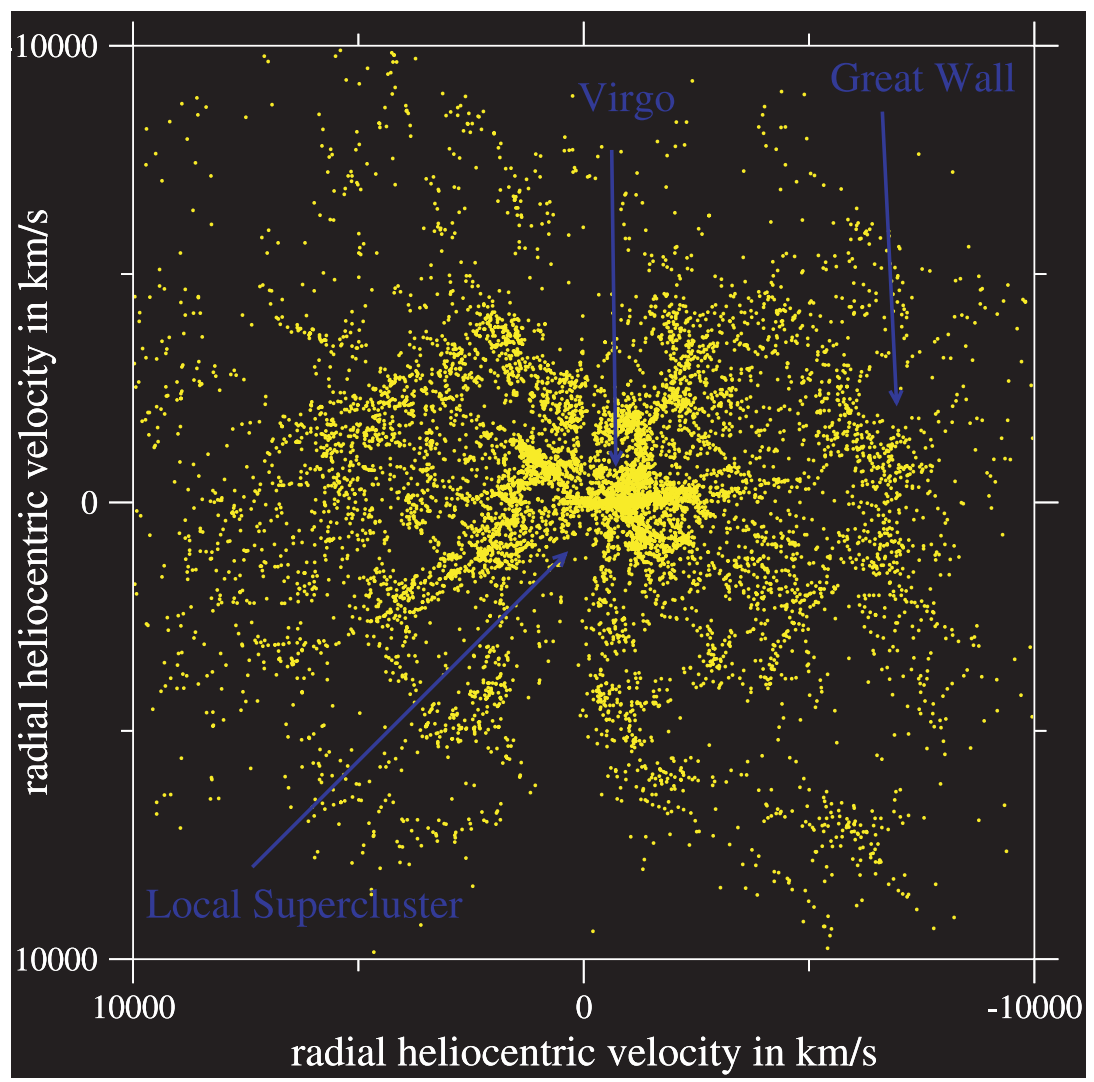

Fig. 8. View face-on of the $X Z$ plane in supergalactic coordinates, slice of $50 \mathrm{Mpc}$ along the $Y$ axis. 


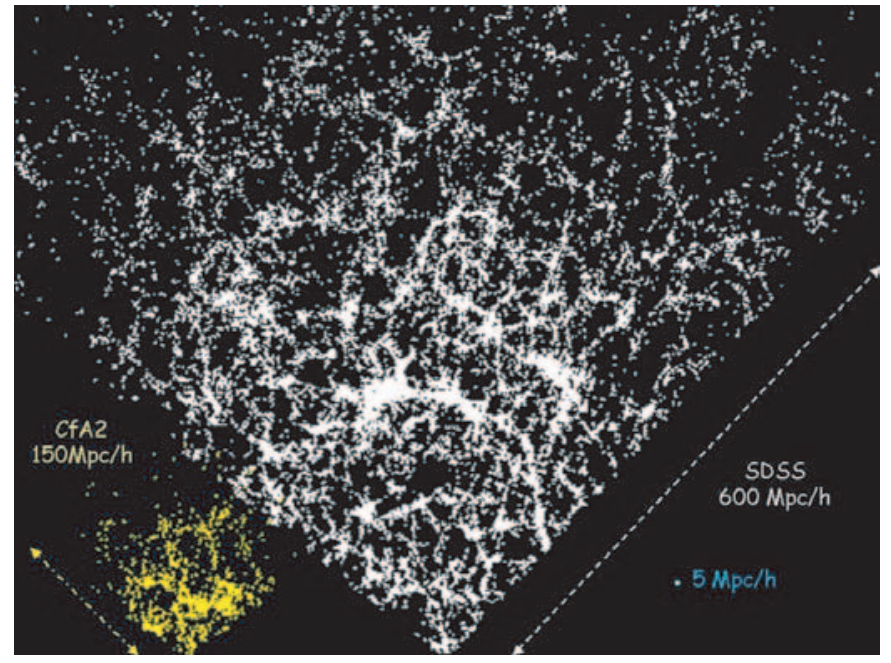

Fig. 9. Comparison between the first public data release of SDSS and CFA2.

It is striking to see that the very local view of the local universe with bubbles, chains and walls can still be seen at a scale 10 times larger. One cannot guess the intrinsic scale of the structures by looking at these maps. We can really begin to speak about hierarchy in the large-scale structures, another word for scale invariance. Studies of the statistical properties of such distributions are ongoing and we will present them soon. For the moment, it is for instance clear that a galaxy correlation-length of $15 \mathrm{Mpc}$ could hardly be a meaningful statistical indicator tracing the galaxy clustering properties in the Local Universe.

\section{Conclusions}

With the astonishing large-scale structure observed by 2MASS, SDSS, $2 \mathrm{dF}$ and through the LEDA archive, it is increasingly clear that cosmological models must account for structures ten times larger than previously simulated, going up to $300 \mathrm{Mpc}$ long. We cannot simply estimate the scale at which one is looking at the structure, just by looking at the maps: we are seeing similar structures at different scales. This could be another argument in favor of using general tools that can describe both homogeneous and scale invariant distribution, to analyse the galaxy point distribution.

Acknowledgements. We thank very much the referee, Thomas Jarrett, for his help in improving this paper. F. Sylos Labini acknowledges the support of a Marie Curie Fellowship HPMF-CT-2001-01443.

\section{References}

Abazajian, K., Adelman-McCarthy, J. K., Agüeros, M. A., et al. (The SDSS Collaboration) 2003, AJ, 126, 2081

Amendola, L., Di Nella, H., Montuori, M., \& Sylos-Labini, F. 1997, Fractals, 5, 635

Colless, M., Dalton, G., Maddox, S., Sutherland, W., et al. (2dFGRS team) 2001, MNRAS, 328, 1039

Cutri, R. M., Skrutskie, M. F., Van Dyk, S., et al. 2003, Explanatory Supplement to the 2MASS all sky data release, http://www. ipac. caltech.edu/2mass/releases/allsky/doc/explsu.html

de Vaucouleurs, G., de Vaucouleurs, A., \& Corwin, H. G. 1976, Second reference catalog of bright galaxies (Austin: Texas University Press) (RC2)

de Vaucouleurs, G., de Vaucouleurs, A., Corwin, H. G., et al. 1991, Third reference catalog of bright galaxies (Springer-Verlag) (RC3)

Di Nella, H., \& Paturel, G. 1994, Comptes rendus de l'académie des sciences Paris, Série II, t319, 57

Di Nella, H., \& Paturel, G. 1995, PASA, 12, 26

Di Nella, H., Montuori, M., Paturel, G., Pietronero, L., \& Sylos-Labini, F. 1996, A\&A, 308, L33

Di Nella, H., Parker, Q. A., Couch, W. J., \& Paturel, G. 1997, MNRAS, 287, 472

Geller, M. J., \& Huchra, J. P. 1989, Science, 260, 1175

Huchra, J., \& Burg, R. 1992, ApJ, 393, 90

Huchra, J. P., Geller, M. J., \& Corwin, H. G. Jr. 1995, ApJS, 99, 391

Jarrett, T. H., Chester, T., Cutri, R., et al. 2000, AJ, 119, 2498

Paturel, G., Andernach, H., Bottinelli, L., et al. 1997, A\&AS, 124, 109

Paturel, G., Bottinelli, L., Di Nella, H., Fouqué, P., \& Gouguenheim, L. 1994, A\&A, 289, 711

Schlegel, D. J., Finkbeiner, D. P., \& Davis, M. 1998, ApJ, 500, 525 